

\title{
Col4a1 mutation generates vascular abnormalities correlated with neuronal damage in a mouse model of HANAC syndrome
}

\author{
Alix Trouillet, Henri Lorach, Elisabeth Dubus, Brahim El Mathari, Ivana \\ Ivkovic, Julie Dégardin, Manuel Simonutti, Michel Paques, Xavier \\ Guillonneau, Florian Sennlaub, et al.
}

\section{To cite this version:}

Alix Trouillet, Henri Lorach, Elisabeth Dubus, Brahim El Mathari, Ivana Ivkovic, et al.. Col4a1 mutation generates vascular abnormalities correlated with neuronal damage in a mouse model of HANAC syndrome. Neurobiology of Disease, 2017, 100, pp.52 - 61. 10.1016/j.nbd.2016.12.014 . hal-01436672

\section{HAL Id: hal-01436672 \\ https://hal.sorbonne-universite.fr/hal-01436672}

Submitted on 16 Jan 2017

HAL is a multi-disciplinary open access archive for the deposit and dissemination of scientific research documents, whether they are published or not. The documents may come from teaching and research institutions in France or abroad, or from public or private research centers.
L'archive ouverte pluridisciplinaire HAL, est destinée au dépôt et à la diffusion de documents scientifiques de niveau recherche, publiés ou non, émanant des établissements d'enseignement et de recherche français ou étrangers, des laboratoires publics ou privés. 
Col4a1 mutation generates vascular abnormalities correlated with neuronal damage in a mouse model of HANAC syndrome

Running title: Retinal damage in Col4a1 mutated mice

Alix Trouillet ${ }^{1,2,3,11}$, Henri Lorach ${ }^{1,2,3,11}$, Elisabeth Dubus ${ }^{1,2,3}$, Brahim El Mathari ${ }^{4}$, Ivana Ivkovic $^{1,2,3}$, Julie Dégardin ${ }^{1,2,3}$, Manuel Simonutti ${ }^{1,2,3}$, Michel Paques ${ }^{1,2,3,5}$, Xavier Guillonneau $^{1,2,3}$, Florian Sennlaub ${ }^{1,2,3}$, José-Alain Sahel ${ }^{1,2,3,5,6,7}$, Pierre Ronco ${ }^{8,9,10}$, Emmanuelle Plaisier ${ }^{8,9,10^{*}}$, and Serge Picaud ${ }^{1,2,3^{*}}$.

${ }^{1}$ INSERM, U968, Institut de la Vision, Paris, F-75012, France ;

${ }^{2}$ Sorbonne Universités, UPMC Univ Paris 06, UMR_S968, Institut de la Vision, Paris, F75012, France;

${ }^{3}$ CNRS UMR7210, Institut de la Vision, Paris, 75012, France ;

${ }^{4}$ Fovea Sanofi, Paris, France;

${ }^{5} \mathrm{CHNO}$ des Quinze-Vingts, Paris ;

${ }^{6}$ Fondation Ophtalmologique Adolphe de Rothschild, Paris, France ;

${ }^{7}$ Academie des Sciences, Paris.

${ }^{8}$ AP HP, Department of Nephrology, Tenon Hospital, Paris, France ;

${ }^{9}$ INSERM, U1155, Paris, F-75020, France ;

${ }^{10}$ Sorbonne Universités, UPMC Univ Paris 06, UMRS_1155, Paris, F-75020, France ;

${ }^{11}$ Stanford University, CA, USA

* These two authors contributed equally to the work

$\underline{\text { Key Words: }}$ Collagen, retinal damage, mouse model, vascular permeability 


\section{Corresponding authors:}

Emmanuelle Plaisier

Department of Nephrology,

Bâtiment Recherche,

Hôpital Tenon,

4 rue de la Chine,

75020 Paris

France

Tel. +33156016513

Email.emmanuelle.plaisier@tnn.aphp.fr

Serge Picaud, PhD

Institut de la Vision

17 rue Moreau

75012 Paris

France

Tel. +33153462592

Email.serge.picaud@inserm.fr

\section{Summary Statement}

This study provides a phenotypic analysis of a novel mouse model of HANAC syndrome focusing on the retinal aspect. It recapitulates most of the aspects of the human disease and is therefore a great tool to study and to address this condition. 


\section{Abstract}

The HANAC syndrome is caused by mutations in the gene coding for collagen4a1, a major component of blood vessel basement membranes. Ocular symptoms include an increase in blood vessel tortuosity and occasional hemorrhages. To examine how vascular defects can affect neuronal function, we analyzed the retinal phenotype of a HANAC mouse model. Heterozygous mutant mice displayed both a thinning of the basement membrane in retinal blood vessels and in Bruch's membrane resulting in vascular leakage. Homozygous mice had additional vascular changes, including greater vessel coverage and tortuosity. This greater tortuosity was associated to higher expression levels of vascular endothelial growth factor (VEGF). These major changes to the blood vessels were correlated with photoreceptor dysfunction and degeneration. The neuronal damage was associated with reactive gliosis in astrocytes and Müller glial cells, and by the migration of microglial cells into the outer retina. This study illustrates how vascular changes can trigger neuronal degeneration in a new model of HANAC syndrome that can be used to further study dysfunctions of neurovascular coupling. 


\section{INTRODUCTION}

Although changes in the neurovascular coupling of the retina occur in many retinal diseases, it often remains unclear whether neurons are affected before major vascular changes, such as leakage, tortuosity and hemorrhages, occur (Barber et al., 2011). Hereditary retinal diseases affecting blood vessel structure provide useful models for investigating the sequential events relating vascular changes to neuronal dysfunction. COLAAl mutations have been identified in patients presenting type I porencephaly associated with cerebral small vessel disease (CSVD) (Gould et al., 2005). These brain defects have been associated, in some but not all cases, with various eye abnormalities, including ocular anterior segment dysgenesis, juvenile-onset glaucoma, cataract, microphthalmia, and, more rarely, retinal arteriolar tortuosities (Coupry et al., 2010; Deml et al., 2014; Sibon et al., 2007). A distinctive phenotypic presentation of COL4Al-related diseases has been characterized: HANAC, an acronym for Hereditary Angiopathy, Nephropathy, Aneurysms and muscle Cramps. HANAC is associated with systemic symptoms, such as kidney disease and muscle cramps, a form of CSVD less severe than previously reported, single or multiple brain aneurysms. Unlike porencephaly, HANAC is consistently characterized by bilateral retinal arteriolar tortuosity associated with repeated retinal hemorrhages (Plaisier et al., 2005; Plaisier et al., 2007), whereas no other ocular abnormalities, particularly those affecting the anterior segment of the eye in porencephaly, have been reported.

The COLAA1 gene encodes the $\alpha 1$ chain of collagen IV, which assembles with the $\alpha 2$ chain to form the most abundant basement membrane protein, the $\alpha 1 \alpha 1 \alpha 2$ collagen IV heterotrimer (Kuo et al., 2012; Mayne et al., 1984; Trueb et al., 1982). The collagenous domain, a long stretch of Gly-Xaa-Yaa repeats forming a triple helix, accounts for over $80 \%$ of the protein. The autosomal dominant $C O L 4 A 1$ mutation responsible for HANAC affects a 30-amino acid segment of this collagenous domain located between residues Gly498 and 
Gly528 and containing an integrin-binding site, whereas the mutations associated with the phenotype restricted to the brain and eye generally affect the C-terminal half of the COL4A1 protein, suggesting a possible genotype-phenotype correlation (Plaisier et al., 2010). Interestingly, a recent study linked familial retinal arteriolar tortuosity (FRAT) to a missense mutation p.Gly510Arg in COL4A1 (Zenteno et al., 2014), a mutation previously identified in HANAC patients. For the first time, the molecular basis of FRAT was identified showing that identical mutations in COL4A1 can lead to both systemic and eye-restricted phenotypes (Zenteno et al., 2014).

The role of COLAA1 mutations in porencephaly was discovered in a random embryonic stem cell mutagenesis study aiming to identify the genes contributing to glaucoma, a disease causing blindness through retinal ganglion cell loss and optic nerve hypoplasia. The isolated Col4a1 $1^{+/ 4 \operatorname{ex} 40}$ mutant mouse line displayed not only ocular anterior segment dysgenesis and buphthalmos (large eyes), but also porencephaly with cerebral hemorrhages (Gould et al., 2005). All animals had clinical symptoms of anterior segment dysgenesis, such as corneal opacification, iris pigment dispersion, iridocorneal synechiae, cataracts, persistence of the tunica vasculosa lentis and abnormal iris vasculature (Gould et al., 2007). This dysgenesis lead to glaucoma with a loss of one third of the retinal ganglion cells and the consecutive optic nerve hypoplasia detected as early as postnatal day 10 (Gould et al., 2007). Other mutations in the Gly-Xaa-Yaa repeats were discovered in parallel to induce a similar ocular phenotype as the Col4al $^{+/ 4 \text { ex40 }}$ mutation (Van Agtmael et al., 2005).


because no abnormal phenotype was observed in mice with a null allele (Gould et al., 2005).

Here we developed a new model of HANAC syndrome bearing the Col4al pGly498Val substitution homologous to the human COLAA1 pGly498Val mutation responsible for the HANAC phenotype in one family. The eye is a particularly interesting 
model to investigate the neurovascular coupling because photoreceptors are among the most energy consuming cells and we investigated here the retinal phenotype in this mouse model of HANAC syndrome.

\section{RESULTS}

\section{Vascular tortuosity}

All patients suffering from HANAC syndrome display retinal arteriolar tortuosity and occasional retinal hemorrhages. We therefore began our analysis of mutant Col4al G498V mice by examining the retinal vascular network at three and nine months of age. The retina was collected and immunolabeled with an anti-collagen IV antibody, for reconstruction of the entire vascular network (Fig. 1A-B). A visual inspection of the flat-mounted retinas revealed the presence of abnormally tortuous structures in homozygous mice (Fig. 1C-D) and these vascular modifications were quantified (Fig. 1E-J). Significantly higher levels of retinal coverage by the vessel network were found in both heterozygous and homozygous mutant mice than in wild-type mice at three months. By nine months, this difference was no longer significant in heterozygous animals, but retinal coverage by the vessel network remained $24.5 \%$ higher in homozygous than in WT mice (Fig. 1H). Consistent with the increase in blood vessel coverage, total vessel length was also $25 \%$ greater in homozygous mutant mice than in WT mice (Fig. 1G). By contrast, no significant difference in this parameter was observed between heterozygous and WT animals at either ages. Retinal vascular tortuosity, the landmark of HANAC syndrome in patients, was quantified specifically, by determining the number of branching points and mean squared vessel curvature (Hart et al., 1999). Homozygous mutant mice had $30 \%$ more branching points than WT mice, a significant difference, at the ages of three and nine months (Fig. 1I). The squared vessel curvature in homozygous mice was almost twice that of the WT mice at both three and nine months (Fig. 
1J). No significant differences in these parameters were observed between heterozygous and WT mice (Fig. 1G-J). Thus, despite the changes in the vascular plexus already visible at three months of age and persisting thereafter in homozygous mice, the milder changes in heterozygous mice, as indicated by the higher rate of coverage at three months, seemed to resolve as the animal aged.

\section{Vascular leakage}

To investigate vascular function, we assessed blood vessel leakage in vivo using the scanning laser ophthalmoscope (SLO) (Fig. 2A-B). Under fluorescein angiography, clear images of dye leakage were observed at 10 months in some homozygote animals (white arrow, Fig. 2B). To quantify this blood vessel leakage, we used the Evans blue permeation method. In nine-month-old animals, this test indicated that retinal vascular permeability was 1.75 times greater in mice homozygous for the mutant Col4al allele than in WT animals (Fig. 2C). Surprisingly, despite the absence of vascular changes observed during morphological analysis of the vascular plexus, a similar and significant increase in retinal vessel permeability was detected in heterozygous mice (Fig. 2C). This finding suggests that Col4al haploinsufficiency or the mutated protein induces a dysfunction of the blood-retina barrier. An increase in retinal vessel permeability is often associated with an increase in VEGF expression (Kurihara et al., 2014). We therefore investigated the level of expression of the genes encoding VEGF and its receptors in the retina. In homozygous Col4al mutant mice, VEGF-A mRNA levels were higher than those of WT mice at nine months, whereas no difference was found between heterozygous and WT mice (Fig. 2D). No difference was observed in the expression of the VEGF receptor R1 between mutant and WT mice, whereas VEGF R2 mRNA levels were significantly higher in homozygous mutant mice than in the WT (Fig. 2D). Thus, there was a major change in the functioning of the blood-retina barrier, 
even in heterozygous animals, which was not associated with an increase in VEGF levels in heterozygous mice, whereas an increase in these levels was observed in homozygous mutant mice.

\section{Ultrastructure of the retinal vessel basement membrane}

We investigated the cause of the rupture of the blood-retina barrier, by first examining the retinal vessel basement membrane in both homozygous and heterozygous Col4al mutant mice. Ultrastructure analysis showed that the basement membrane surrounding the retinal blood vessels was very thin (Fig. 2E-F), with many focal interruptions, in Col4al mutant mice (black arrows). The basement membrane was $40 \%$ thinner in both homozygous and heterozygous mutant mice than in WT mice (Fig. 2G). We then examined the retinal Bruch's membrane, which together with retinal pigment epithelium (RPE) cells comprises the outer blood retinal barrier (Curcio and Johnson, 2013). Its thickness in Col4al homozygous mutant mice appeared strongly reduced at the ultrastructural level (Fig $2 \mathrm{H}-\mathrm{I})$ with a $33 \%$ reduction when compared to control animals (Fig. 2J). In addition, the number of NG2-immunopositive pericytes was decreased by $20 \%$ (Ho: $18.9+/-0.2$ cells/per microscope field; $n=2$ and WT: $24+$ +- 0.39/per microscope field; $n=2$ ) in homozygous mice. These different observations could potentially account for the greater vascular permeability in mutant mice.

\section{Neuronal alterations}

We investigated whether the blood vessel tortuosity and abnormal vascular permeability observed in Col4al mutant mice affected retinal function. We recorded electroretinograms (ERGs), to assess retinal function. By three months of age, the amplitude of the scotopic ERG a-wave, indicative of rod photoreceptor function, was lower in homozygous mutant mice than in WT animals at three and nine months, whereas this wave 
was normal in heterozygous mice (Fig. 3A-B). The lower b-wave ERG amplitudes under scotopic conditions in homozygous mice at both ages confirmed the functional deficit (Fig. 3C). Under photopic conditions and $10 \mathrm{~Hz}$ flickers, providing functional information about the cone pathway (Fig. 3D), ERG amplitudes were significantly lower in homozygous mutant mice than in WT animals at nine months, but not at three months (Fig. 3E-F). Interestingly, in heterozygous mice, none of the ERG parameters measured differed significantly from the values obtained for WT mice. These results suggest that both the rod and cone pathways are affected by the vascular deficit already evident at the age of three months but only in homozygous mutant animals, and the dysfunction seems to concern the rods in the rod pathway. We assessed the fate of the retinal tissue further, by examining the retina in vivo by optical coherence tomography (OCT) scanning (Fig. 4A-B). The lens was clear, with no sign of anterior segment dysgenesis or buphthalmos, allowing correct visualization of the retinal tissue. At the ages of both three and nine months, morphological changes were observed, with a significantly lower total retinal thickness in homozygous mutant mice (Fig. 4C). Layer-by-layer analyses on OCT scans showed an absence of change in the thickness of the outer nuclear layer (ONL) formed by the nuclei of the photoreceptors (data not shown), whereas the layer corresponding to the outer and inner segments of the photoreceptors was significantly thinner (12\% thinner) in homozygous mutant mice (Fig. 4D) as well as the area corresponding to the inner plexiform layer (IPL) and ganglion cell layer (GCL) (14\% thinner, Fig. 4D). Immunostaining was performed on retinal sections, to determine the type of cell responsible for this decrease in retinal thickness. Immunolabeling of the cone photoreceptors with the cone arrestin antibody revealed signs of alteration to cone photoreceptor outer/inner segments (Fig. 5A-D). Cone degeneration worsened with aging, with a $30 \%$ decrease in cone density at nine months (Fig. 5M). The rod photoreceptor dysfunction was accompanied by an abnormal shape of rod bipolar cells immunolabeled with 
a PKC $\alpha$ antibody (Fig. 5E-H). These neurons were already shorter than those of the WT mice at the age of three months (Fig. 5E-F), and they appeared disorganized at nine months (Fig. 5G-H). Quantification of retinal ganglion cell (RGC) number was performed using antiBrn3a antibody (Fig. 5I-L) showing a slight but not significant decrease in RGC number in mutant animals compared to WT (Fig. 5N). In this experiment, Brn3a, which is a transcription factor, was used as a specific cell marker of RGCs because it is expressed in more than $92 \%$ of RGCs and RGCs are the only retinal cells expressing Brn3a (NadalNicolas et al., 2009; Schlamp et al., 2013). A 30\% decrease in the number of retinal ganglion cells has been shown to be associated with the considerable variability of intraocular pressure (IOP) measurements for Col4al ${ }^{+/ \Delta \mathrm{ex} 40}$ mice (Gould et al., 2007). We therefore measured IOP in our mutant mice (Fig. 5O). IOP values were also highly dispersed in the homozygous mice, but mean values were similar between genotypes. These observations indicate that Col4al G498V mutant mice do not present glaucoma, with its increase in IOP and associated loss of retinal ganglion cells.

Therefore, the neuronal dysfunctions and damage in homozygous mutant mice principally concern the photoreceptors.

\section{Gliosis and the microglial reaction}

Neuronal damage is often associated with inflammation and gliosis. In the retina, neurodegeneration and inflammation are associated to reactive gliosis, characterized by the activation of astrocytes as well as Müller cells (Fernandez-Sanchez et al., 2015). Those macroglial cells of the retina have also major structural, metabolic and homeostatic roles that are crucial for retinal health. Reactive gliosis induce their secretion of neurotrophic factors as well as cytokines (Bringmann et al., 2006; Harada et al., 2003). A classic retinal marker for reactive gliosis is the upregulation of glial fibrillary acidic protein (GFAP) expression in glial 
Müller cells. In WT animals, GFAP is expressed in astrocytes and in the Müller cell endfeet (Fig. 6A). On retinal cross sections, GFAP immunolabeling was much stronger in homozygous mutant mice than in controls, with the GFAP distribution in the Müller cell processes extending to the outer plexiform layer (Fig. 6B). On flat-mounted retina stained with GFAP (Fig. 6D-I), the astrocyte network appeared to be denser in nine-month-old homozygous mutant animals than in WT animals. The staining was much stronger around blood vessels than elsewhere (arrows in Fig. 6I). Quantification of the coverage of the retina by the astrocyte network indicated 20 and $35 \%$ greater coverage in nine-month-old heterozygous and homozygous mutant mice, respectively, than in control animals (Fig. 6C). No significant difference was observed at three months, suggesting an increase in gliosis over time.

We also assessed microglial cell activity by labeling retinal sections with anti-Iba1 and anti-TSPO antibodies. Iba1 is a protein with actin and calcium binding sites. This protein, which expression is enhanced by cytokine, interleukin and interferon, can regulate cellular events such as proliferation, migration and phagocytosis through its interaction with actin cytoskeleton (Imai and Kohsaka, 2002). In neuronal tissue, Iba1 is specifically expressed in microglial cells such that it can be used to specifically label these cells to examine their morphology and their localization. When microglia are activated in pathological conditions, Iba1 can enable us to document microglial morphological changes and their migration providing thereby a classic marker of brain injury and disease.

In mutant homozygous mice, we detected microglial activation, characterized by a change in the cellular shape of the cells already detectable at the age of three months. Resting microglial cells are star-shaped and are surrounded by long processes (Fig. 7A, C, blue arrow, G, I), whereas activated microglial cells become ameboid and lose their extensions (white arrow in Fig. 7B, D, and H, J). Non-activated microglial cells are classically found in the 
outer and inner plexiform layers, whereas activated microglial cells were found in the photoreceptor nuclear layer and even in photoreceptor outer segments at nine months (Fig. 7D, white arrow). The microglial activation was confirmed by the induced drastic expression of TSPO (Fig. 7E-F), a known marker of inflammation in the mammalian retina (Karlstetter et al., 2014; Wang et al., 2014). Interestingly, activated microglial cells in the mutant animals did not specifically migrate towards the blood vessels but remained homogeneously spread across the retina as shown by flatmount double staining of blood vessels and Iba1 (Fig. 7GH). We quantified Iba1 mRNA levels throughout the retina and found that Iba1 mRNA levels were $60 \%$ higher in homozygous mice than in control mice (Fig. 7K). These observations confirmed the pathological state of the neural retina.

\section{DISCUSSION}

We showed, in a murine model of HANAC syndrome, that a mutation of the gene encoding for the Col4a1 protein, a major component of the basement membrane, was responsible for a strong vascular phenotype in the retina, associated with neuronal degeneration in homozygous mutant animals. Mutant animals displayed a significant increase in vessel tortuosity and length as early as 3 months of age. Vascular leakage could be punctually observed in some mutants, and vascular permeability was almost doubled in homozygous mice. This was associated to an increased in VEGF-A and VEGF-R2 mRNA expression and a thinning of basement and Bruch's membrane. A strong visual phenotype was revealed by ERG impairment in both scotopic and photopic conditions associated to morphological changes such as photoreceptor degeneration, bipolar cell modifications, and inflammation throughout the retina. This first viable model of homozygous Col4a1 mutant mice reproduces the retinal vascular involvement observed in patients with HANAC syndrome. Although HANAC is an autosomal dominant condition in human, the homozygous model 
displays a more robust and severe retinal phenotype. The recent study linking FRAT and the COL4A1 G510R mutation (Zenteno et al., 2014) demonstrated that environmental and/or genetic factors might influence the phenotypic expression and the severity of the disease (Chen et al., 2015; Guiraud et al., 2016). This report corroborates the heterogeneity found in the heterozygote mutant mice, and justifies the use of the homozygous mice with robust phenotype to study the molecular basis of HANAC syndrome. This mutant model would also allow the study of more general neurovascular defects. The mutant animals displayed no ocular anterior segment dysgenesis or optic nerve hypoplasia, as opposed to Col4al and Col4a2 ENU mutant mice, which bear mutations different from those associated with the HANAC phenotype in patients (Gould et al., 2007; Gould et al., 2005; Kuo et al., 2014; Labelle-Dumais et al., 2011; Van Agtmael et al., 2005). This observation suggests that the pathogenic effect of the mutations responsible for HANAC syndrome may differ at least partly from those associated with purely brain- and eye-restricted phenotype. Indeed, in addition to the retinal phenotype, the COL4A1 G498V mutation was also associated with renal and muscle defects similar to those observed in HANAC patients (Chen et al., 2015; Guiraud et al., 2016).

Evans blue staining revealed the occurrence of vascular leakage in both homozygous and heterozygous mutant mice. Surprisingly, although this vascular permeability was associated with higher Vegf-a mRNA levels in homozygous animals, as reported in many pathological conditions (Kurihara et al., 2014), no similar increase in VEGF-A levels was observed in heterozygous animals. These results suggest that the levels of VEGF-A expression observed cannot by itself account for the retinal vascular leakage. Instead, the increased vascular permeability in mutant mice may be due to the thinning of the basement membranes and is consistent with the role of collagen IV in ensuring its mechanical resistance. This thinning in the basement membrane is also consistent with the defective 
secretion of the mutant $\alpha 1 \alpha 1 \alpha 2$ (IV) protein that is abnormally retained in the endoplasmic reticulum as observed in muscles of the HANAC animal model (data not shown).

However, the vascular leakage by itself, affecting both heterozygous and homozygous mutant mice does not seem to be responsible for the visual dysfunction as it only occurs in homozygous animals. Conversely, the strong vascular remodeling that includes abnormal vessel tortuosity, increase in vessel length and density is already significant at the age of three months in homozygous animals only, and occurs concomitantly with visual defects.

This neuronal dysfunction did not result from a loss of retinal ganglion cells during development as opposed to the observations on $\operatorname{Col}_{4 a 1^{+/ 4 e x 40}}$ mice (Gould et al., 2007). Instead, the ERG recordings indicated a photoreceptor defect and were correlated to cone loss, as demonstrated by cell counting. This cone degeneration could be the result of a dysfunction in the basement membrane at either the retinal blood vessels or the Bruch's membrane. These retinal barriers are involved in the metabolic support to photoreceptors, including oxygen, nutrients, electrolytes, cytokines, vitamins and signaling molecules allowing the proper cellular activity (Ahmed et al., 1993). Among neurons that already rank first among cells for energy consumption, photoreceptors are responsible for the consumption of the bulk part of retinal oxygen and glucose under conditions of darkness (Wong-Riley, 2010). Dysfunction in this metabolic support is very likely to induce photoreceptor degeneration as in the case of mutations expressed in choroidal capillaries (Kennan et al., 2005) but could also involve toxicity of the mutant protein as described in the Col4al $^{+/ 4 e x 40}$ mice (Gould et al., 2005). The neuronal alterations were accompanied by a large increase in GFAP expression in Müller glial processes, a marker of retinal lesions (Lewis and Fisher, 2003), as well as a greater astrocytic coverage. Further evidence for the induction of neuronal lesions was provided by the increase in microglial cell density in retinal tissues. In patients, retinal tortuosity is associated with hemorrhages although no vascular leakage was reported, 
as indicated by fluorescein angiography results (Plaisier et al., 2005; Plaisier et al., 2007). Those individuals experience episodic transient losses of vision due to these occurrences of retinal hemorrhages but the visual prognosis of HANAC patients remains excellent, without retinal sequelae (Plaisier et al., 2005; Plaisier et al., 2007). These retinal hemorrhages may reveal complete ruptures of the blood-retina barrier. The thinning of the vascular basement membrane reported here in both heterozygous and homozygous animals is consistent with the weakening of the vascular wall and high probability of hemorrhages in affected patients if blood pressure is high. Furthermore, such weakening of the vascular basement membrane could account not only for its complete rupture, but also for its partial rupture, leading to fluid extravasation into the retinal tissue, resulting to swelling, as observed in macular edema (Gardner and Antonetti, 2008). Anti-VEGF therapies can attenuate such edemas, but their persistence in many patients may reflect other underlying mechanisms, leading to partial rupture of the blood-retina barrier (Elman et al., 2011; Nguyen et al., 2012). The absence of increase in VEGF-A levels in heterozygous Col4al-mutant mice, despite the permeation, is consistent with this observation. Further studies are required to determine whether the physical thinning of the vascular basement membrane is sufficient to trigger these extravasations or whether they involve more specific molecular mechanisms. Our Col4al mutant mice may therefore prove to be an appropriate model for studies of blood-retina barrier permeation independent of increases in VEGF-A expression.

The Col4a1 G498V mutant mouse is a relevant model for HANAC syndrome, reproducing defects observed in patients, not only in the retina but also in kidneys (Chen et al., 2015) and in muscles (Guiraud et al., 2016). Further studies in this model could also determine whether hyperoxia or continuous light exposure could limit neuronal dysfunction and blood vessel tortuosity, as proposed for other vascular diseases such as diabetic retinopathy (Arden et al., 2011; Frost-Larsen, 1991; Kurtenbach et al., 2006). More generally, 
the Col4a1 G498V mutant mice provide new insight in the retinal pathophysiology of HANAC syndrome retinal defects.

\section{MATERIALS AND METHODS}

\section{Animals}

The Col4al G498V mouse line was established at the MCI/ICS in a C57BL/6 background (Chen et al., 2015). These animals were tested and found negative for mutations frequently encountered in breeding colonies including rd8 (Crb1 gene), rd1 (Pde6b gene) and cpfl3 (Gnat2). Mice were housed in cages with a $12 \mathrm{~h}$ dark/light cycle, with food and water available ad libitum. All experiments were carried out in accordance with European Community Council Directives (86/609/EEC) and with the ARVO (Association for Research in Vision and Ophthalmology) statement for the use of animals in ophthalmic and visual research.

\section{SD-OCT imaging}

Images were acquired on the SD-OCT imaging device (Bioptigen $840 \mathrm{~nm}$ HHP; Bioptigen, North Carolina, USA) on anesthetized animals. The thickness of retinal layers was determined manually $500 \mu \mathrm{m}$ from the center of the optic nerve.

\section{Scanning Laser Ophthalmoscope imaging}

Photographs of the eye fundus and angiographs were obtained with a scanning laser ophthalmoscope (SLO) (HRA, Heidelberg, Germany). Fluorescein retinal angiography was carried out after the intraperitoneal injection of $25 \%$ fluorescein in $0.9 \% \mathrm{NaCl}$ and pupils were dilated by tropicamide eye drops (0.5\%) (Paques et al., 2006). 


\section{Electroretinography}

Light was provided by a $150-\mathrm{W}$ xenon lamp in a Ganzfeld stimulator (Multiliner Vision; Jaeger/Toennies, Hochberg, Germany), at 3 candela (cd)/s/m². Photopic cone ERGs were performed with a rod-suppressing background light of $25 \mathrm{~cd} / \mathrm{m}^{2}$, after a 5 -minute adaptation. Responses were amplified and filtered (1 Hz-low and $300 \mathrm{~Hz}$-high cutoff filters) with a onechannel DC-/AC-amplifier. Each response shown is the mean of five responses to flashes. Flicker light stimulation was performed at $10 \mathrm{~Hz}$ and responses were averaged over a $40 \mathrm{~s}$ stimulation period.

\section{Histology and immunohistochemistry}

Cryosections $(12 \mu \mathrm{m})$ or flat-mounted retinas were incubated with primary antibody against cone arrestin (Rabbit polyclonal antibody, 1/10000; \#AB15282, Merck-Millipore), PKC alpha (Rabbit polyclonal Antibody, 1/1000; \#sc-208, Santa Cruz Biotechnology), Brn3a (Mouse Monoclonal Antibody, 1/100; \#MAB1585, Merck Millipore), Iba1 (Rabbit polyclonal Antibody, 1/500; \#W1W019-19741, Sobioda, Wako), TSPO (Rabbit monoclonal antibody, 1/250; \#Ab109497, Abcam), Collagen IV (Goat anti Human Collagen IV, 1/200; \#134001, AbD Serotec), NG2 (Rabbit polyclonal Antibody, 1/200; \#AB5320, Merck Millipore), and glial fibrillary acidic protein (GFAP, Mouse Monoclonal Antibody, 1/1000; \#G3893-.2ML) to visualize cones, bipolar cells ganglion cells, microglial cells, the vascular network, both astrocytes, pericytes and Muller glial cells, respectively. Secondary goat antirabbit IgG or goat anti-mouse IgG conjugated to either Alexa TM594 or Alexa TM488 were applied. Cell nuclei were revealed incubating the specimens with 4', 6-diamidino-2phenylindole (DAPI) and images obtained using an Olympus Fluoview1000 confocal microscope.

The vascular plexus was extracted to calculate the number of branching points, the total length of the vasculature and square curvature, as described in a previous study (Hart et al., 
1999). The GFAP coverage factor was calculated as the ratio of the GFAP-labeled area to the retinal explant area. Pericytes counting has been performed on total retinal flat mount.

\section{Quantification of blood-retina barrier permeability}

Vascular permeability was quantified by measuring albumin leakage from blood vessels into the retina by the Evans blue method (Xu et al., 2001). We injected Evans blue (45 mg/kg; Sigma-Aldrich, Germany) through the penile vein of anesthetized mice. Blood samples were taken $3 \mathrm{~h}$ after injection. Mice were perfused with citrate buffer $\left(0.05 \mathrm{M}, \mathrm{pH} 3.5,37^{\circ} \mathrm{C}\right)$ for 2 minutes via the left ventricle. Retinas were carefully dissected and dried, weighed, and Evans blue was extracted before centrifugation at $16873 \mathrm{~g}$ for $2 \mathrm{~h}$ (retinas) or 15 minutes (blood) at $4^{\circ} \mathrm{C}$. After measuring absorbance, the concentrations of Evans blue in the plasma and retina were calculated from a standard curve of Evans blue in formamide. Blood-retina barrier permeability was then calculated, in microliters of Evans blue per gram of wet retina per hour $\left(\mu 1\right.$ Evans blue $\times \mathrm{g}$ wet retina $\left.{ }^{-1} \times \mathrm{h}^{-1}\right)$.

\section{Ultrastructure examination}

Mice were perfused with $4 \%$ PFA $+2 \%$ glutaraldehyde in cacodylate buffer $(\mathrm{pH}=7.4)$ after anesthesia. The eyes were enucleated, and incubated in this solution. Retinas were then cut into small pieces and post-fixed in osmic acid $\left(1 \% \mathrm{OsO}_{4}\right.$ in cacodylate buffer) for $1 \mathrm{~h}$. After dehydration, samples were embedded and cut with an ultramicrotome and contrasted with $2 \%$ uranylacetate and Reynold's lead citrate (Reynolds, ES (1963)) before observation with a Hitachi HT7700 electron microscope. 


\section{Quantitative PCR analysis}

RNA was extracted from total retina using Nucleopsin RNA XS Kit XS RNA (\#740902, Macherey-Nagel) and converted to cDNA by reverse transcription using SuperscriptII (Invitrogen). Quantitative Real Time PCR (RT-PCR) were performed in presence of 200nM of specific primers for each genes (VEGF A, VEGF R1, VEGF R2 and Iba1), $200 \mu \mathrm{M}$ dNTP (Invitrogen), $1.5 \mathrm{mM} \mathrm{MgCl}_{2}$ and 1.0 U Taq DNA polymerase (Invitrogen). All real-time data were normalized with respect to ATP6.

\section{Acknowledgments}

We would like to thank Dr. Dominique Langui from the EM platform at Pitié-Salpétrière Hospital, and Dr. Fouquet and David Godefroy from the imaging platform at the Vision Institute for expert assistance in data acquisition. We thank Fovea Pharmaceuticals (SANOFI) for technical support.

\section{Competing interests}

The authors declare no conflicting interest.

\section{Authors contributions:}

AT, EP, SP designed the study. PR, EP designed the mouse line. AT, ED, II, JD, MS

collected the data. AT, HL, BM, MP, XG, FS analyzed the data. AT, SP wrote the paper. HL, ED BM, II, JD, MS, MP, XG, FS, JS, PR, EP critically revised and approved the manuscript. JS, SP obtained funding. 
References

Ahmed, J., et al., 1993. Oxygen distribution in the macaque retina. Invest Ophthalmol Vis Sci. 34, 516-21.

Arden, G. B., et al., 2011. Regression of early diabetic macular oedema is associated with prevention of dark adaptation. Eye (Lond). 25, 1546-54.

Barber, A. J., et al., 2011. The significance of vascular and neural apoptosis to the pathology of diabetic retinopathy. Invest Ophthalmol Vis Sci. 52, 1156-63.

Bringmann, A., et al., 2006. Muller cells in the healthy and diseased retina. Prog Retin Eye Res. 25, 397-424.

Chen, Z., et al., 2015. HANAC Syndrome Col4a1 Mutation Causes Neonate Glomerular Hyperpermeability and Adult Glomerulocystic Kidney Disease. J Am Soc Nephrol.

Coupry, I., et al., 2010. Ophthalmological features associated with COL4A1 mutations. Arch Ophthalmol. 128, 483-9.

Curcio, C. A., Johnson, M., Chapter 20 - Structure, Function, and Pathology of Bruch's Membrane. In: S. J. R. R. S. R. H. P. S. R. S. P. W. W. P. Schachat, (Ed.), Retina (Fifth Edition). W.B. Saunders, London, 2013, pp. 465-481.

Deml, B., et al., 2014. Whole exome analysis identifies dominant COL4A1 mutations in patients with complex ocular phenotypes involving microphthalmia. Clin Genet.

Elman, M. J., et al., 2011. Expanded 2-year follow-up of ranibizumab plus prompt or deferred laser or triamcinolone plus prompt laser for diabetic macular edema. Ophthalmology. 118, 609-14.

Fernandez-Sanchez, L., et al., 2015. Astrocytes and Muller Cell Alterations During Retinal Degeneration in a Transgenic Rat Model of Retinitis Pigmentosa. Front Cell Neurosci. 9, 484.

Frost-Larsen, K., 1991. Macular recovery recorded by nyctometry in insulin-dependent diabetes mellitus. Acta Ophthalmol Suppl. 1-39.

Gardner, T. W., Antonetti, D. A., 2008. Novel potential mechanisms for diabetic macular edema: leveraging new investigational approaches. Curr Diab Rep. 8, 263-9.

Gould, D. B., et al., 2007. Col4a1 mutation causes endoplasmic reticulum stress and genetically modifiable ocular dysgenesis. Hum Mol Genet. 16, 798-807.

Gould, D. B., et al., 2005. Mutations in Col4a1 cause perinatal cerebral hemorrhage and porencephaly. Science. 308, 1167-71.

Guiraud, S., et al., 2016. HANAC Col4a1 mutation in mice causes a muscular disease with endoplasmic reticulum stress and vascular defects. The American journal of pathology. In press.

Harada, C., et al., 2003. Potential role of glial cell line-derived neurotrophic factor receptors in Muller glial cells during light-induced retinal degeneration. Neuroscience. 122, 229-35.

Hart, W. E., et al., 1999. Measurement and classification of retinal vascular tortuosity. Int J Med Inform. 53, 239-52.

Imai, Y., Kohsaka, S., 2002. Intracellular signaling in M-CSF-induced microglia activation: role of Iba1. Glia. 40, 164-74.

Karlstetter, M., et al., 2014. Translocator protein (18 kDa) (TSPO) is expressed in reactive retinal microglia and modulates microglial inflammation and phagocytosis. J Neuroinflammation. $11,3$.

Kennan, A., et al., 2005. Light in retinitis pigmentosa. Trends Genet. 21, 103-10.

Kuo, D. S., et al., 2012. COL4A1 and COL4A2 mutations and disease: insights into pathogenic mechanisms and potential therapeutic targets. Hum Mol Genet. 21, R97-110.

Kuo, D. S., et al., 2014. Allelic heterogeneity contributes to variability in ocular dysgenesis, myopathy and brain malformations caused by Col4a1 and Col4a2 mutations. Hum Mol Genet. 23, 1709-22. 
Kurihara, T., et al., 2014. Hypoxia-inducible factor (HIF)/vascular endothelial growth factor (VEGF) signaling in the retina. Adv Exp Med Biol. 801, 275-81.

Kurtenbach, A., et al., 2006. Hyperoxia, hyperglycemia, and photoreceptor sensitivity in normal and diabetic subjects. Vis Neurosci. 23, 651-61.

Labelle-Dumais, C., et al., 2011. COL4A1 mutations cause ocular dysgenesis, neuronal localization defects, and myopathy in mice and Walker-Warburg syndrome in humans. PLoS Genet. 7, e1002062.

Lewis, G. P., Fisher, S. K., 2003. Up-regulation of glial fibrillary acidic protein in response to retinal injury: its potential role in glial remodeling and a comparison to vimentin expression. Int Rev Cytol. 230, 263-90.

Mayne, R., et al., 1984. Monoclonal antibodies against chicken type IV and V collagens: electron microscopic mapping of the epitopes after rotary shadowing. J Cell Biol. 98, 1637-44.

Nadal-Nicolas, F. M., et al., 2009. Brn3a as a marker of retinal ganglion cells: qualitative and quantitative time course studies in naive and optic nerve-injured retinas. Invest Ophthalmol Vis Sci. 50, 3860-8.

Nguyen, Q. D., et al., 2012. Ranibizumab for diabetic macular edema: results from 2 phase III randomized trials: RISE and RIDE. Ophthalmology. 119, 789-801.

Paques, M., et al., 2006. High resolution fundus imaging by confocal scanning laser ophthalmoscopy in the mouse. Vision Res. 46, 1336-45.

Plaisier, E., et al., 2005. Autosomal-dominant familial hematuria with retinal arteriolar tortuosity and contractures: a novel syndrome. Kidney Int. 67, 2354-60.

Plaisier, E., et al., 2010. Novel COL4A1 mutations associated with HANAC syndrome: A role for the triple helical CB3[IV] domain. Am J Med Genet A.

Plaisier, E., et al., 2007. COL4A1 mutations and hereditary angiopathy, nephropathy, aneurysms, and muscle cramps. N Engl J Med. 357, 2687-95.

Schlamp, C. L., et al., 2013. Evaluation of the percentage of ganglion cells in the ganglion cell layer of the rodent retina. Mol Vis. 19, 1387-96.

Sibon, I., et al., 2007. COL4A1 mutation in Axenfeld-Rieger anomaly with leukoencephalopathy and stroke. Ann Neurol. 62, 177-84.

Trueb, B., et al., 1982. Basement membrane (type IV) collagen is a heteropolymer. J Biol Chem. 257, 5239-45.

Van Agtmael, T., et al., 2005. Dominant mutations of Col4a1 result in basement membrane defects which lead to anterior segment dysgenesis and glomerulopathy. Hum Mol Genet. 14, 31618.

Wang, M., et al., 2014. Macroglia-microglia interactions via TSPO signaling regulates microglial activation in the mouse retina. J Neurosci. 34, 3793-806.

Wong-Riley, M. T., 2010. Energy metabolism of the visual system. Eye Brain. 2, 99-116.

$\mathrm{Xu}, \mathrm{Q}$., et al., 2001. Sensitive blood-retinal barrier breakdown quantitation using Evans blue. Invest Ophthalmol Vis Sci. 42, 789-94.

Zenteno, J. C., et al., 2014. Next generation sequencing uncovers a missense mutation in COL4A1 as the cause of familial retinal arteriolar tortuosity. Graefes Arch Clin Exp Ophthalmol. 252, 1789-94. 


\section{FIGURE LEGENDS}

Figure 1. Vascular plexus remodeling in the homozygous mutant mice. (A-D) Flat-mounted retinas immunolabeled with collagen IV antibody, for control (WT) animals (A \& C) and homozygous (Ho) mutant mice (B \& D). Note the tortuosity of the retinal vessels in the homozygous mutant mice (arrows in D). (E-J) Quantification of the vascular plexus paremeters at 3 and 9 months, in WT animals (black dot), heterozygous (He, gray square) and Ho (gray triangle) mutant mice. All vascular parameters (vessel length $(\mathrm{G})$, vessel coverage $(\mathrm{H})$, number of branches (I) and squared curvature (J)) were already significantly increased in Ho mutant mice as compared to WT mice at three months. Means \pm SEM are shown $* p<0.05, * * p<0.01, * * * p<0.005$ vs. the control group (Two way ANOVA and Turkeys comparison) 
Figure 2. Increase in retinal vascular permeability associated to variable VEGF expression and thinning of the blood vessel basement membrane in Col4al mutant mice. (A-B) Fluorescein retinal angiography showing vascular leakage (B - white arrow) in homozygous animal at 10 months. (C) Measurement of vascular permeability in the Evans blue-albumin permeation test in control animals (WT, $\mathrm{n}=15$ ), heterozygous (He, $p=0.0004, n=18$ ) and homozygous (Ho $p=5 \times 10^{-5}, n=10$ ) Col4al mutant retinas. (D) Expression of VEGF-A and its receptors (VEGF-R1 and VEGF-R2), as assessed with total retinal mRNA, for WT animals $(n=3)$, and for $\mathrm{He}(n=4)$ and Ho $(n=3)$ mutant mice $(p<0.05)(\mathrm{E}-\mathrm{F})$ Ultrastructure of the retinal pericyte basement membrane (BM) in control (WT) animals (E) and in homozygous (Ho) mutant mice $(\mathrm{F})$. Note the thinning of the basement membrane (black arrows ) and the presence of focal interruptions in the Ho mouse (white arrow in F). (G) Basement membrane thickness in WT animals (20 measurements) and in heterozygous (He, 15 measurements) and Ho (15 measurements) Col4al mutant mice. Ultrastructure of the Bruch's membrane (BrM) in control (WT) animals (H) and in Ho mutant mice (I). Bruch's membrane thickness is reduced by half in He and Ho mutant mice compared to WT animals (J). *** $p<0.005$ vs. the control group (one-way ANOVA plus Turkeys comparison ). Scale bar represents $200 \mu \mathrm{m}$ in $(\mathrm{C}-\mathrm{D})$ and $800 \mu \mathrm{m}$ in $(\mathrm{F}-\mathrm{G}) . \quad * * * p<0.005$ and $* * * * p<0.0005$ vs. the control group ( $t$-tests). Scale bar represents $200 \mu \mathrm{m}$ in $(\mathrm{E}-\mathrm{F})$ and $800 \mu \mathrm{m}$ in $(\mathrm{H}-\mathrm{I})$. 
Figure 3. Functional deficit of the retina in Col4al mice. (A) Representative scotopic electroretinogram (ERG) recordings for a control (WT) animal and a homozygous (Ho) Col4al mutant mouse at 9 months. (B-C) Quantification of a-wave (B) and b-wave amplitudes (C) under scotopic conditions 3 candela $(\mathrm{cd}) / \mathrm{s} / \mathrm{m}^{2}$, at 3 and 9 months, in WT animals, heterozygous (He) and Ho Col4al mutant mice. (D) Photopic ERG recordings at 9 months in a WT animal and a Ho mouse. (E-F) Quantification of the photopic ERG (E) and the flicker response amplitudes (F) at 3 and 9 months in WT, He and Ho mice. Each response shown is the mean of five responses to flashes. $* p<0.05$, ** $p<0.01, * * * p<0.005$ vs. the control group (two-way ANOVA plus Dunn's comparison). 
Figure 4. OCT retinal imaging in Col4al mutant mice. (A-B) OCT scans in a control (WT A) animal and a homozygous (Ho-B) Col4al mutant mouse at 9 months. (C) Thickness of the total retina at 3 and 9 months old, (D) of the photoreceptor inner/outer segment layer (IS+OS) and the inner plexiform layer and ganglion cell layer (IPL+GCL) a 9 months in WT and Ho mutant animals. ${ }^{*} p<0.05, * * p<0.01, * * * p<0.005, * * * * p<0.001$ IS: Inner Segment, OS : Outer Segment, IPL : Inner Plexiform Layer, GCL : Ganglion Cell Layer. 
Figure 5. Neuronal damage in Col4al mutant mice. (A-J) Retinal sections immunolabeled with a cone arrestin antibody (A-D), with a PKC $\alpha$ antibody identifying rod bipolar cells (EH) and a brn3a antibody identifying retinal ganglion cells (I-L) in control (WT) animals (A, C, E, G, I,K) and in homozygous (Ho) Col4al mutant mice (B, D, F, H, J,L) at 3 months (AB, E-F, I-J) and 9 months (C-D, G-H,K-L). Note the morphological alterations to the cone photoreceptors in Ho mice at 3 months (B) and 9 months. (M-N) Quantification of cone photoreceptors $(\mathrm{M})$ and retinal ganglion cells $(\mathrm{N})$ in WT animals $(n=5)$ and Ho mice $(n=5)$ at 9 months. (O) Intraocular pressure (IOP) measurements in WT animals $(n=12)$, heterozygous (He, $n=12)$ and Ho $(n=6)$ Col4al mutant mice at 9 months. $\quad * p<0.05$ vs. the control group ( $t$-tests M-N); one-way ANOVA and Dunn's comparison (O).. Scale bar represents $100 \mu \mathrm{m}$ in (A-D), $50 \mu \mathrm{m}$ in $(\mathrm{E}-\mathrm{H}), 50 \mu \mathrm{m}$ in $(\mathrm{I}-\mathrm{J})$. 
Figure 6. Glial reaction in Col4al mutant mice. Retinal cross-sections (A-B) and flatmounted retinas (D-I) immunolabeled with a GFAP antibody from control (WT) animals (A, D, G) heterozygous (He-E) and homozygous (Ho) Col4al mutant mice (B, F, H, I) at 9 months. Blood vessels G-H) are surrounded by astrocytes as emphasize in high magnification image (I). (C) Quantification of astrocyte density on flat-mounted retinas from WT animals ( $n=4$ at 3 months, $n=7$ at 9 months), and heterozygous (He: $n=4$ at 3 months, $n=15$ at 9 months) and Ho mutant mice ( $n=4$ at 3 months, $n=6$ at 9 months). $* p<0.05$ one-way ANOVA and Dunn's comparison). Scale bar represents $50 \mu \mathrm{m}$ in (A-B), $200 \mu \mathrm{m}$ in (C-D), $500 \mu \mathrm{m}$ in (E). 
Figure 7. Microglial activation in homozygous Col4al mutant mice. (A-F) Retinal sections immunolabeled with Iba1 and TSPO antibodies to visualize normal and activated microglial cells in control (WT) animals (A, C, E) and homozygous (Ho) Col4al mutant mice (B, D, F), at 3 months (A, B) and 9 months (C-F). Microglial cell activation in the mutant mice is indicated by the morphological change from a cell with long processes to a bulging cell body (white arrow in B, D), by active migration into the photoreceptor layer (white arrow in D), and by the upregulation of TSPO (F). (G-J) Flat-mounted retinas of control (WT) (G, I) and homozygous (Ho) mutant mice $(\mathrm{H}, \mathrm{J})$ at 9 months immunolabeled with both Col4a1 and Iba1 antibodies. Higher magnifications (I-J) show the activation of microglial cells with bulged cell bodies in the homozygous mice $(\mathrm{J})$. (K) Quantification of Iba1 mRNA levels in retinal tissues from WT animals and Ho mice at 9 months. Relative levels were calculated by the comparative $\triangle \Delta \mathrm{Ct}$ method, with the ATP6 gene used for normalization. WT: $n=7$; Ho: $n=7$. *** $p<0.005$ vs. control group ( $t$-tests). ONL: Outer Nuclear Layer, INL: Inner Nuclear


in $\mathrm{C}$ and $\mathrm{F} 30 \mu \mathrm{m}$ in $(\mathrm{C}-\mathrm{F}, \mathrm{I}-\mathrm{J})$. 

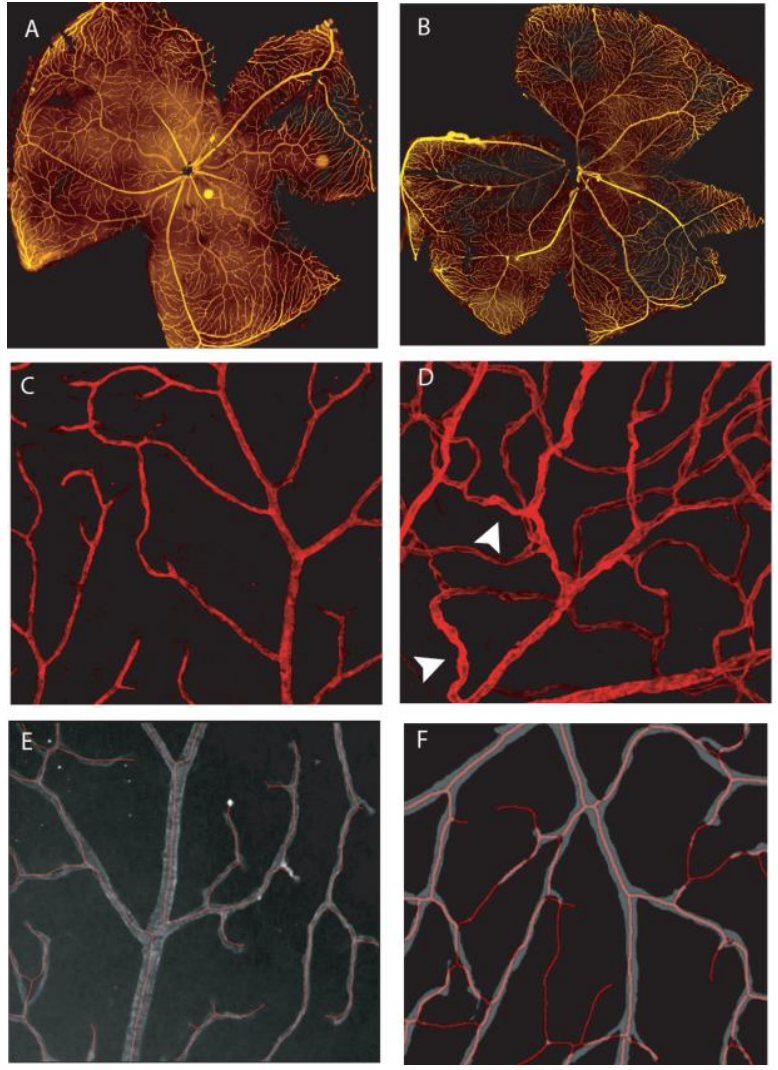

Fig. 1
- $W T=\mathrm{He} \wedge \mathrm{Ho}$

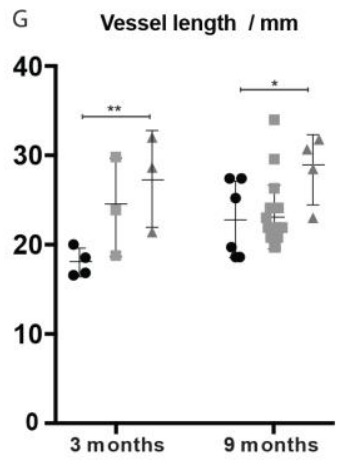

H Vessel coverage factor

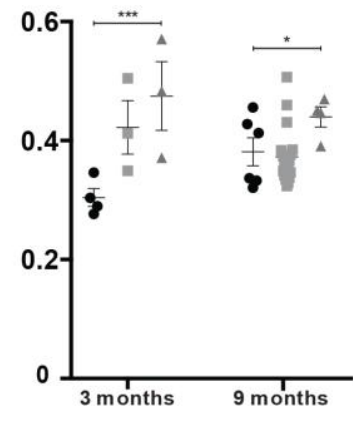

I Number of branches $/ \mathrm{mm}^{2}$
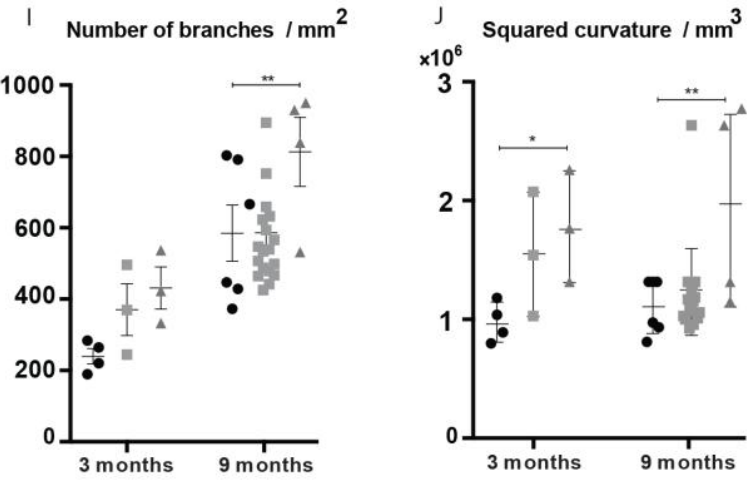

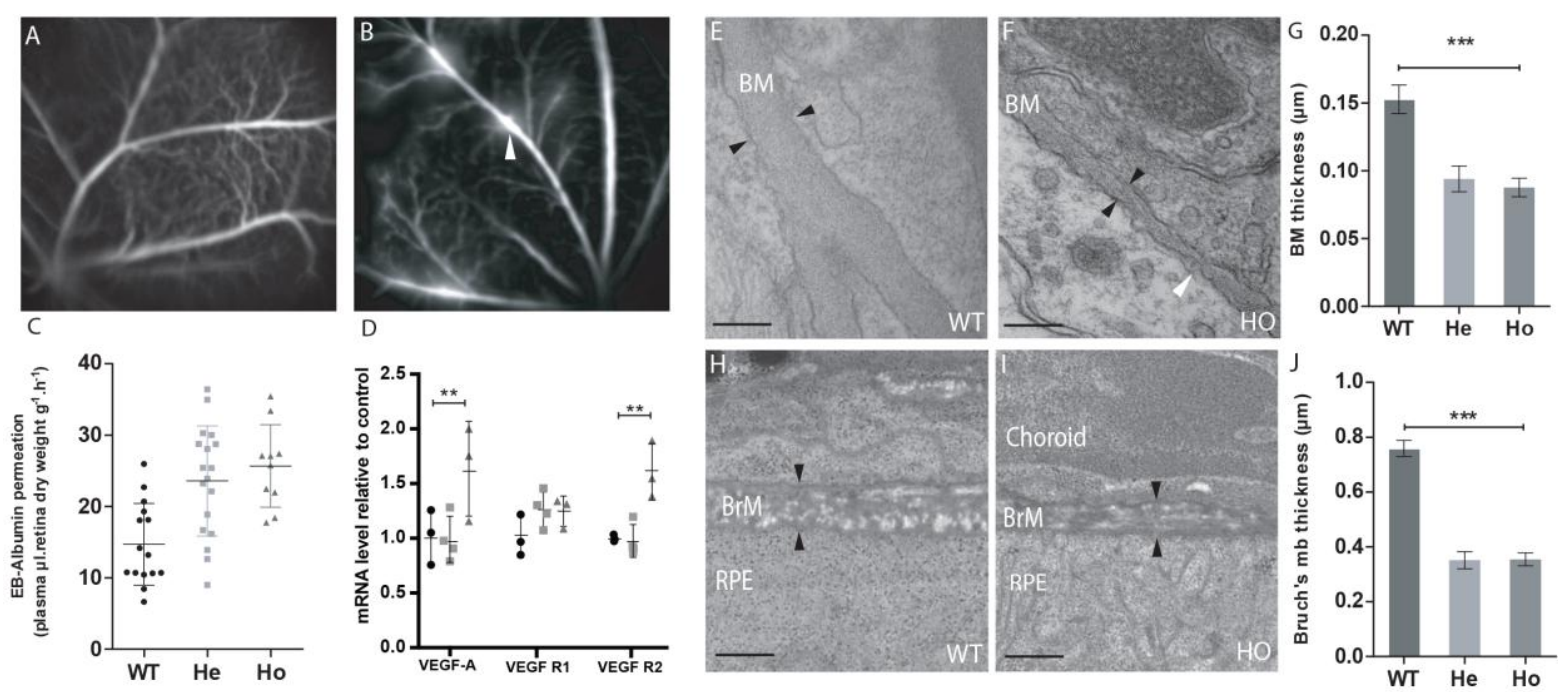

Fig. 2 

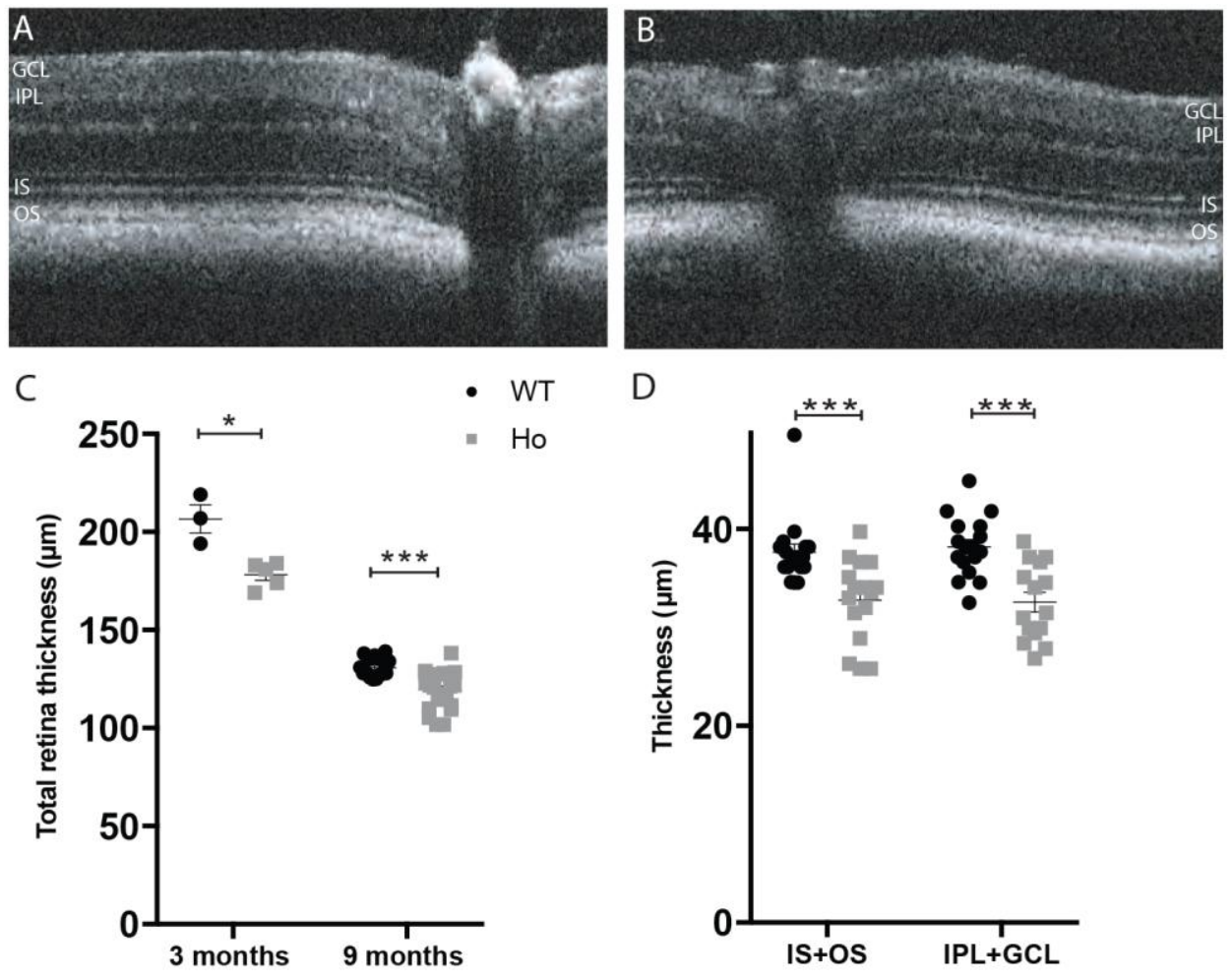

Fig. 3 
A


D

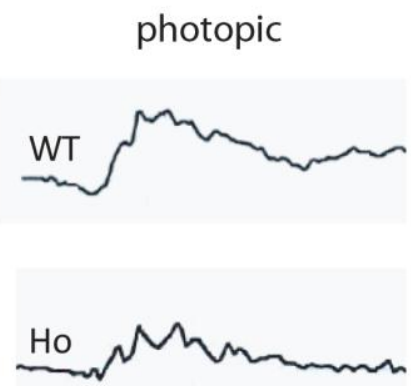

B

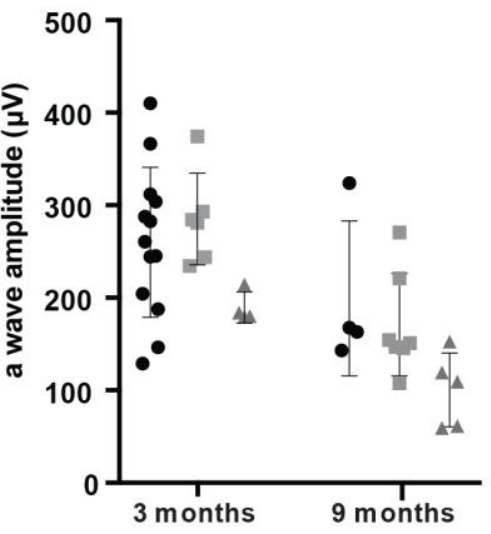

E

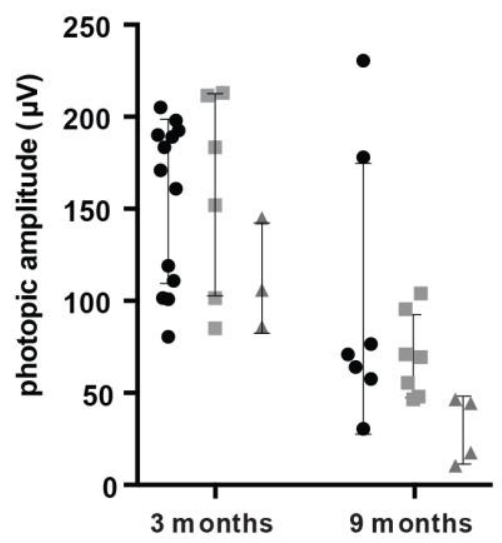

C



F

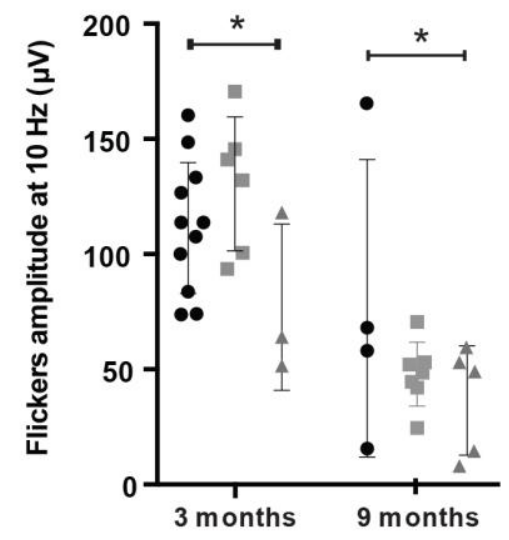

Fig. 4 


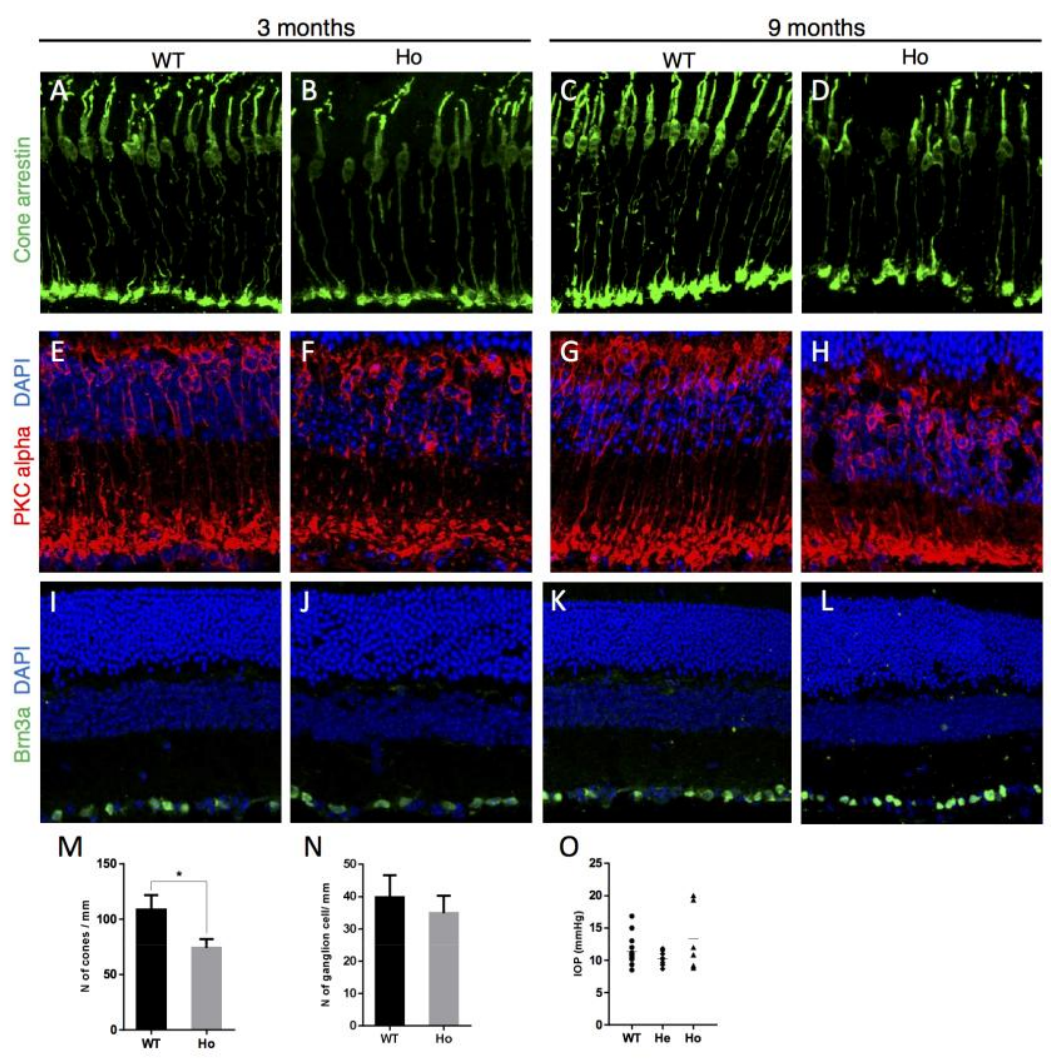

Fig. 5 


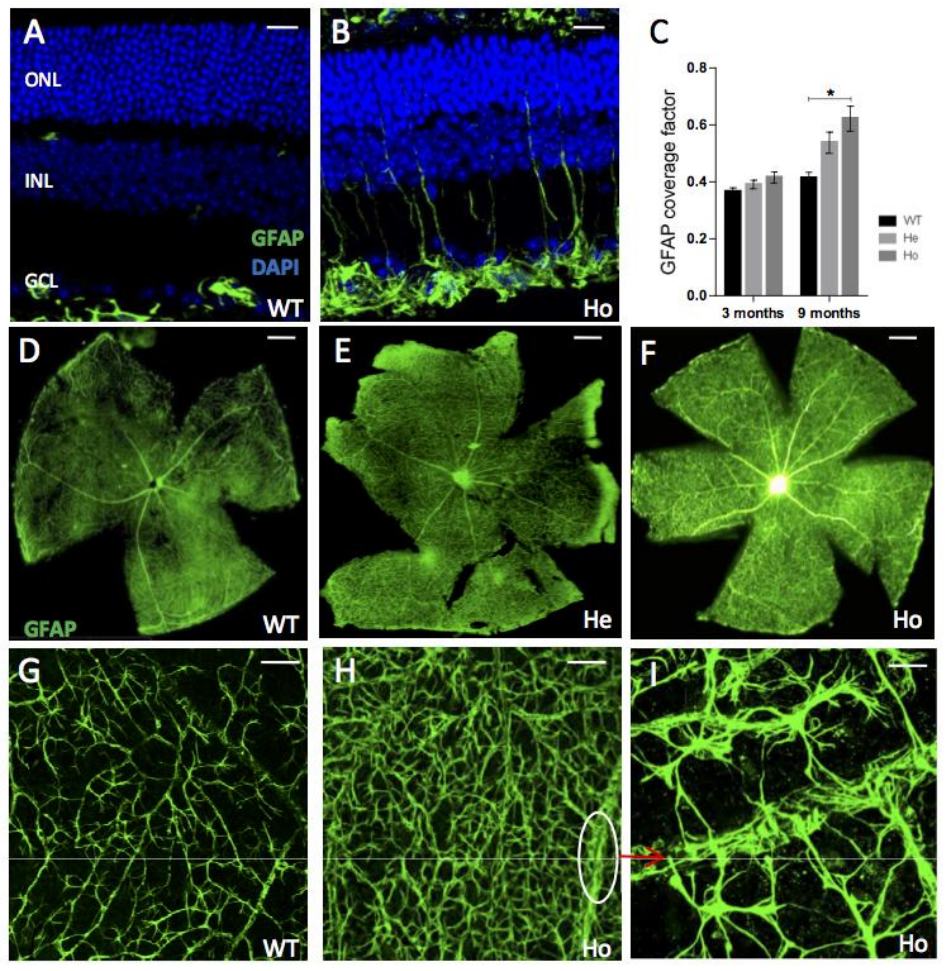

Fig. 6 

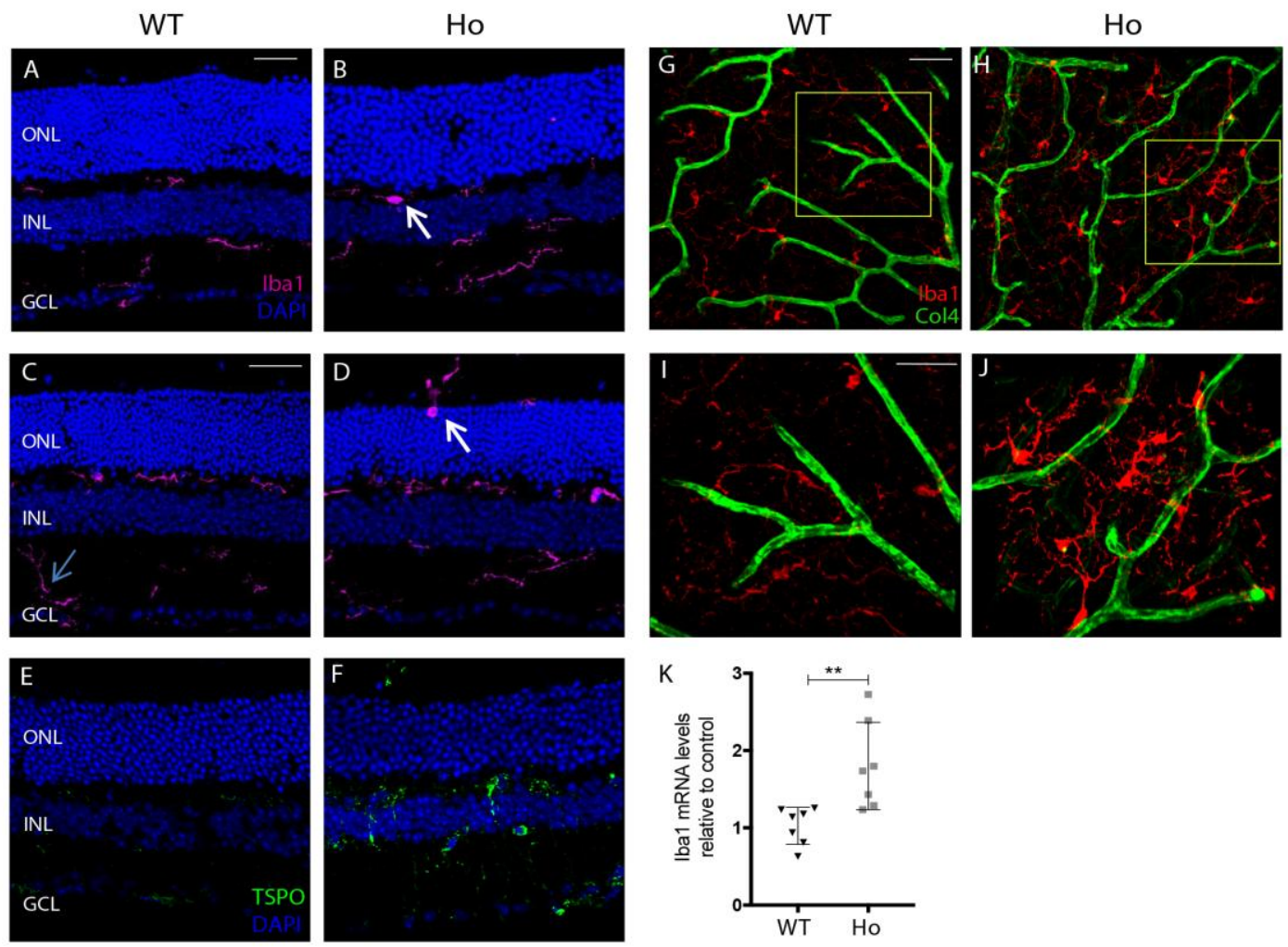

Fig. 7 
Highlights

- We develop and characterize the visual phenotype of a mouse model of HANAC syndrome

- It mimics the human pathology in particular vessel tortuosity and vascular leakage

- Neuronal dysfunction associated with these vascular abnormalities is characterized

- This model is well suited to investigate the neurovascular coupling in HANAC syndrome 